\title{
Métodos físicos e químicos para o tratamento de efluentes
}

\author{
Suéllen Tonatto Ferrazzo, Débora Ferasso, Marluci Marangoni, \\ Mateus Velho dos Santos, Orlando de Paris Junior, Taís Carla \\ Gaspareto, Gean Delise Leal Pasquali Vargas
}

Universidade Federal da Fronteira Sul. Programa de Pós-Graduação em Ciência e Tecnologia Ambiental - PPGCTA. ERS 135, km 72, 200. Caixa Postal 764. Erechim-RS, Brasil (CEP 99700-970). E-mail: suellenferrazzo@hotmail.com.

Resumo. A busca por processos de tratamento de efluentes que sejam eficientes e garantam a segurança à saúde pública e ao meio ambiente tem sido um grande desafio nas últimas décadas, devido à presença de microrganismos persistentes e micropoluentes orgânicos e inorgânicos que resistem aos tratamentos convencionais. Assim, as tecnologias alternativas de tratamento de efluentes vêm sendo aplicadas em associação aos tratamentos primários e secundários, para alcançar maiores reduções da carga de poluentes, utilizando menor espaço físico e consumo de energia elétrica, bem como menor produção de lodo. Neste trabalho, buscou-se realizar um estudo da arte através de uma revisão dos métodos físicos e químicos para o tratamento de efluentes: membranas de filtração, eletrocoagulação, adsorção, troca iônica, processos oxidativos avançados, ozonização e precipitação química, considerando o princípio de funcionamento suas vantagens, desvantagens e aplicações de cada técnica.

Palavras-chave: Efluentes; Métodos físicos e químicos; Tecnologias alternativas.

Abstract. Physico-chemical methods for the treatment of effluents. The search for effluent treatment processes that are efficient and guarantee safety to public health and the environment has been a great challenge in the last decades due to the presence of persistent microorganisms and organic and inorganic micropollutants that resist conventional treatments. Thus, alternative technologies for effluent treatment have been applied in association with primary and secondary treatments, to achieve greater reductions in pollutant load, using less space and electrical energy consumption, as well as lower sludge production. In this work, a study of the art was made through a review of the physical-chemical methods for the treatment of effluents: filtration membranes, electrocoagulation, adsorption, ion exchange, advanced oxidative processes, ozonization and chemical precipitation, considering the principle of advantages, disadvantages and applications of each technique.
Recebido:

19/11/2018

Aceito:

29/12/2018

Publicado:

31/12/2018

Acesso aberto

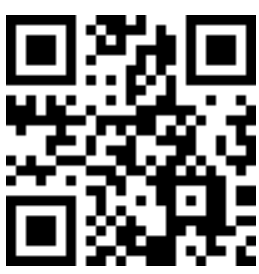

ORCID

(1) 0000-0003-3413-4747

Suéllen Tonatto

Ferrazzo

() 0000-0003-0218-3093

Débora Ferasso

(1) 0000-0001-5908-6193

Marluci Marangoni

(D) 0000-0002-6762-3435

Mateus Velho dos Santos

(1) 0000-0002-1656-9459 Orlando de Paris Junior

(1) 0000-0002-9187-0081 Taís Carla Gaspareto

(1) 0000-0001-5110-6532

Gean Delise Leal

Pasquali Vargas 
Keywords: Wastewater; Physical-chemical methods; Alternative technologies.

\section{Introdução}

O lançamento de efluentes sem tratamento adequado ou parcialmente tratado, em corpos receptores, representa uma potencial fonte de contaminação ambiental, pois estas águas residuárias podem conter elevada carga orgânica e nutrientes, hidrocarbonetos, metais pesados e compostos traços, potencializando processos de eutrofização e problemas de ordem sanitária, ambiental e econômica (Marcogliese et al., 2015; Regan, 2016; Uslu et al., 2016; Ben et al., 2017; Edokpayi et al., 2017; Noor et al., 2017). 0 tratamento primário e secundário de efluentes é capaz de eliminar em torno de $90 \%$ a $99,9 \%$ dos microrganismos entéricos. Enquanto que, os processos de tratamento terciário possibilitam novas reduções, onde podem-se eliminar populações resistentes aos tratamentos iniciais, como vírus, esporos bacterianos, cistos de protozoários (Rafraf et al., 2016; Uslu et al., 2016) além de, micro poluentes orgânicos como produtos farmacêuticos, biocidas e pesticidas (Ben et al., 2017; El-Taliawy et al., 2017).

Realizar o tratamento de águas residuárias que garanta $o$ atendimento aos padrões ambientais e consequentemente, segurança ao seu lançamento no corpo receptor, é um desafio, pois requer a integração de diferentes processos, considerando as características específicas de cada efluente e a viabilidade sob o ponto de vista técnico e econômico (Abdel-Raouf et al., 2012). Neste contexto, é importante avaliar as tecnologias convencionais e inovadoras para 0 tratamento de efluentes, buscando equilibrar a economia do processo com a sustentabilidade ambiental, por meio de reduções na área requerida, no consumo de eletricidade, na geração de lodo e redução ou até eliminação da carga de poluentes (Alvarino, 2018).

0 presente artigo consiste em uma revisão das tecnologias alternativas para tratamentos físico-químicos de efluentes, membranas de filtração, eletrocoagulação, adsorção, troca iônica, processos oxidativos avançados, ozonização e precipitação química, abordando as características, limitações e aplicações de cada processo, com o intuito de avaliar as características de cada processo e sua potencialidade de uso no tratamento de efluentes.

\section{Tecnologias para tratamento de efluentes}

\section{Membranas de filtração}

As membranas de filtração são consideradas uma das tecnologias mais promissoras para o tratamento e a reutilização de águas, destacando-se por permitirem a separação seletiva de partículas, área de projeto compacta e com simples operação, fornecendo águas com qualidade superior em relação as resultantes dos tratamentos convencionais (Kumar et al., 2013; Aguiar et al., 2016; Bengani-Lutz et al., 2017; Bortoluzzi et al., 2017). A aplicação das membranas de filtração, inicialmente utilizada para dessalinização da água salgada, disseminou-se para o tratamento e reutilização de águas residuárias e recuperação de materiais com alto valor agregado, como metais nobres (Badruzzaman, 2009; Ricci et al., 2015; Bortoluzzi et al., 2017; Babilas e Dydo, 2018). As membranas de filtração são aplicadas em diferentes sistemas, abrangendo a Microfiltração (M- 0,05 a $10 \mu \mathrm{m}$ ); a Ultrafiltração (UF- 1 a $100 \mathrm{~nm}$ ); a Nanofiltração (NF- 300 a $500 \mathrm{Da}$ ); a Osmose Reversa (OR- poros inferiores a $1 \mathrm{~nm}$ ); a Osmose Direta (OD); a Destilação por Membrana (DM), a 
Eletrodiálise (ED) e o Biorreator de Membrana (BRM).

As membranas vêm sendo amplamente utilizadas, devido sua eficiência na remoção de diferentes compostos, sendo sua formatação escolhida quanto a porosidade determinante na remoção dos compostos. Alguns trabalhos tem relatado o uso de membranas para o tratamentos de efluentes na remoção de gorduras e bactérias do leite (Kumar et al., 2013; Wojtyniak et al., 2016; Samartino et al., 2017; Zielińska e Galik, 2017); no tratamento de águas residuárias provenientes da indústria farmacêutica, têxtil, de biotecnologia, nas refinarias de petróleo e na engenharia de alimentos, no tratamento de águas ácidas com altas concentrações de metais pesados, bactérias, protozoários, vírus e algumas moléculas de origem orgânica, remoção de corantes e $\mathrm{Na}_{2} \mathrm{SO}_{4}$, remoção de óleo, macropoluentes e íons (Kumar et al., 2013; Duong e Chung, 2014; Ricci et al., 2015; Mohammad et al., 2015; Aguiar et al., 2016; Walker e Narbaitz, 2016; Lin et al., 2016; Cingolani et al., 2017; Bengani-Lutz et al., 2017; Andrade et al., 2017b; Liu et al., 2017; Zielińska e Galik, 2017; Aguiar et al., 2018; Hudaib et al., 2018; Juholin et al., 2018).

A osmose reversa também vem despertando o interesse do setor de produção de energia nuclear, após a sua aplicação na Central Nuclear de Fukushima-Daiichi no tratamento de água do mar contaminada com radiação (Combernoux et al., 2017), a mesma apresenta vantagens como menor consumo de energia elétrica e custo operacional, e pouca incrustação (Rastgar et al., 2017; Gao et al., 2018), também é muito utilizada para dessalinização, tratamento de águas residuárias municipais e efluentes no setor energético (Chou et al., 2010; Hickenbottom et al., 2013; Rastgar et al., 2017; Bell et al., 2017; Gao et al., 2018).

A tecnologia de membranas também é utilizada em associação ao tratamento biológico (geralmente, o anaeróbico), através do biorreator de membranas (BRM), possibilitando obter águas residuárias de alta qualidade, menor área útil e menor consumo de energia elétrica. Os BRM convencionais utilizam sistemas de MF e UF e há também, os biorreatores de membrana osmótica (BRMO) que usam a osmose direta (Judd, 2016; Luján-Facundo et al., 2017). Estes sistemas têm sua maior aplicação no tratamento de efluentes municipais e industriais (Judd, 2016; Iorhemen et al., 2017), na remoção de compostos orgânicos traços, como produtos fármacos, agentes patogênicos e íons (Xie et al., 2012; Luján-Facundo et al., 2017).

Ainda, os diferentes sistemas de membranas podem ser utilizados de forma integrada para o tratamento de águas residuárias, com objetivo de remoção de diferentes contaminantes como: nitrogênio amoniacal total e carbono orgânico total, remoção de DQO (Bortoluzzi et al., 2017; Zielińska e Galik, 2017).

As limitações relativas à utilização das tecnologias de membranas estão na polarização da concentração interna que pode provocar uma redução significativa do fluxo de permeado e em alguns casos, a necessidade de utilizar tecnologias de filtração, por pressão hidráulica como a NF e a OR, para recuperar o permeado (Rastgar et al., 2017), as incrustações ou fouling sob a superfície e que está associada a composição das águas residuárias e aos parâmetros operacionais (Andrade et al., 2017a), há o risco de molhar os poros das membranas, aumentando o fluxo do permeado e, consequentemente, reduzindo a taxa de rejeição (Luo e Lior, 2017; Samartino et al., 2017). Em alguns casos como o da eletrodiálise onde os íons da solução aquosa de alimentação atravessam a membrana de permuta iônica devido ao potencial elétrico (Zhao et al., 2016; Babilas e Dydo, 2017), apresenta alto custo operacional e manutenção periódica (Zhao et al., 2016). 
Assim membranas de filtração são consideradas uma tecnologia de elevada modularidade e seletividade de partículas, sendo utilizada no tratamento de águas residuárias domésticas e industriais, destacando-se pelas elevadas remoções da carga orgânica e também, com aplicação no tratamento de drenagem ácida da mineração e de águas contaminadas por radiação, mostrandose versáteis e eficientes quanto à remoção de poluentes.

\section{Eletrocoagulação}

A eletrocoagulação utiliza eletrodos consumíveis (eletrodo de sacrifício) geralmente compostos de ferro ou alumínio para fornecer íons à solução, permitindo que os contaminantes suspensos, emulsionados ou dissolvidos formem aglomerados não sendo necessário a adição de produtos químicos para formação de agentes coagulantes (Al-Shannag, 2015; Fernandes, 2015). Nesse meio, também há a presença de um cátodo, onde em sua superfície, com a hidrólise da água, ocorre a formação de hidróxidos que, combinam-se com os íons metálicos, originando complexos de hidróxidos metálicos. Posteriormente, estes compostos formam aglomerados (coloides) que interagem com a matéria orgânica presente nas águas residuárias, resultando na formação de flocos. Nos eletrodos, há a formação de gases, principalmente de hidrogênio, que induzem a formação de aglomerados e a flutuação dos flocos (Bassala et al., 2017).

A eletrocoagulação é uma tecnologia de tratamento efetiva para vários tipos de águas residuais, tais como: águas residuais municipais (Daneshvar et al., 2006), águas residuais de aves (Yetilmezsoy et al., 2009), águas oleosas (Yang et al., 2015); efluentes da indústria têxtil (Jung et al., 2015), produção de fermento para fabricação de pães (Kobya e Delipinar, 2008), a biodigestão (On, 2015). Como em qualquer processo, durante a eletrocoagulação alguns parâmetros podem interferir na eficiência do processo como o $\mathrm{pH}$ da solução, a densidade da corrente, tempo de tratamento, potencial do eletrodo, concentração dos poluentes, concentração de ânions, condutividade, entre outros (Chen, 2004; Holt et al., 2002). A eletrocoagulação é um método interessante devido sua simplicidade operacional, baixo consumo de energia, menor tempo de retenção, efluente final de alta qualidade e baixa formação de lodo, apresentando maior versatilidade e compatibilidade ambiental em relação a métodos como adsorção, biossorção, precipitação, permuta iónica, osmose reversa (Al-Shannag, 2015; Fernandes, 2015; Garcia-Segura et al., 2017). O processo também apresenta algumas limitações como o fato de os eletrodos serem eletro-dissolvidos sendo necessário que os mesmos sejam constantemente substituídos. Pode ocorre a formação de uma camada de óxido na superfície do cátodo, podendo acarretar na perda de eficiência do processo, além da necessidade de destinação adequada ao lodo eletroquímico formado (Mollah et al., 2001). A tecnologia da eletrocoagulação faz-se interessante, especialmente devido sua simplicidade operacional e baixo consumo de energia, apesar de apresentar algumas limitações, o processo de eletrocoagulação é muito aplicado e eficiente para o tratamento de águas residuárias advindas de diferentes atividades.

\section{Adsorção}

A adsorção é uma operação de tratamento avançada, utilizada para aumentar a qualidade final ou viabilizar o reuso de efluentes. Uma de suas maiores vantagens é a possibilidade de uso de materiais de baixo custo no tratamento desses efluentes (Babaei et al., 2017). Carvões ativados são tradicionalmente empregados como eficiente adsorvente para remover a variedade de poluentes ambientais. Contudo, ele sofre problemas como a causa de turbidez na água tratada 
no efluente, que limitam o seu uso (Babaei et al., 2017). 0 carvão ativado é o mais vulgarmente utilizado na forma granular (carvão ativado granular (GAC)) ou como um pó (carbono ativado em pó (PAC)). Os carvões ativados podem ser produzidos a partir de uma variedade de materiais carbonáceos, incluindo a madeira, o carvão, a lenhina e cascas de coco (Karanfil e Kilduff, 1999). Vários autores têm demonstrado que GAC e PAC é eficientemente removedor de contaminantes orgânicos da água (Snyder et al., 2007; Delgado et al. 2012). PAC oferece a vantagem de fornecer carbono ativado fresco continuamente, uma vez que é fornecido como um produto novo e não reciclado através do processo de tratamento (Snyder et al., 2007). Os carbonos ativados demonstram um grande potencial para a remoção de $\mathrm{CO}$ vestigiais, embora a dose PAC e GAC e a regeneração/substituição são dois parâmetros críticos a ser considerados para uma remoção bem sucedida (Snyder et al., 2007).

Outros adsorventes de baixo custo podem ser utilizados no tratamento de águas residuárias, como: espuma de poliuretano revestida com o biopolímero quitosana $(0,51$ em peso) para remoção de corantes sintéticas, apresentando resultados como eficiência de $100 \%$ de remoção de azul de metileno. A vantagem de se utilizar biopolímeros naturais é que estes materiais apresentam alta capacidade de adsorção, além de serem biodegradáveis e não tóxicos (Centenaro et al., 2017). Ainda, a quitosana tem sido estudada como adsorvente para remoção de amônia em efluentes de aquicultura, alcançando eficiências de remoção de 100\% (Bernardi et al., 2018). Também, tem-se estudos que relatam o uso de materiais adsorventes no tratamento de diversos efluentes como: tratamento de efluentes têxteis com musgo de turfa (Hemmati et al., 2016) e resíduos sólidos de processos da indústria siderúrgica (Amaral et al., 2016); tratamento de efluente do processo de branqueamento de pastas kraf, utilizando cinzas volantes para remoção de matéria orgânica (Gao e Fatehi, 2018) e remoção de cádmio de efluentes da mineração como o uso de nanocompósitos de hidrogel (Fosso-Kankeu et al., 2017). A adsorção por diferentes materiais, tanto para remoção de compostos orgânicos naturais ou sintéticos, prova ser um processo de remoção eficaz e apresenta um passo de tratamento crucial, que minimiza a presença de poluentes nos efluentes. Nesse processo, a adsorção tem numerosas vantagens, aplicabilidade, facilidade de operação, possibilidade de regeneração e reutilização e baixo custo o que o torna atraente do ponto de vista de aplicação na área de tratamento de efluentes.

\section{Troca iônica}

0 tratamento de águas residuárias pelo método de troca catiônica, principalmente as compostas por soluções diluídas, destaca-se por ser eficiente na remoção de íons e pela simplicidade e baixo custo em sua aplicação. Os principais fatores que influenciam este método são: tempo de contato entre o efluente e a resina, temperatura de operação, $\mathrm{pH}$, taxa de fluxo, concentração inicial do efluente e propriedades da resina (Pintar et al., 2001; Valverde et al., 2006). A troca iônica também pode apresentar algumas limitações, dentre elas está o parâmetro $\mathrm{pH}$, que precisa estar ajustado para ocorrer a troca iônica (Martins et al., 2017).

A troca iônica vem sendo utilizada para o tratamento de águas residuárias de moinho (Ortega et al., 2016), remoção de precursores de n-nitrosodimetilamina e trialometanos de águas residuárias municipais (BeitaSandí e Karanfil, 2017).

Em um estudo desenvolvido para se remover nitrito de corpos hídricos, a permuta iônica pode ser observada como uma solução para o tratamento de águas residuais com alta concentração de nitrito (Li et al., 2010). Estudos 
realizados para a remoção de nitratos de águas superficiais por permuta iônica também são os mais baratos, investimentos de capital e operação tem um custo menor quando comparado com o sistema de osmose reversa (Pintar et al., 2001).

O uso da permuta iônica é observado também no estudo com resinas aniônicas, podendo permitir a absorção de um composto pela troca de íons além da adsorção e melhorar sua remoção global de soluções alcalinas. A absorção de compostos ocorre apenas nos locais ativos da resina por adsorção ou permuta iônica. Assim, os mesmos sites de resina são considerados acessíveis para ambos os modos de absorção e, portanto, a proporção da absorção do soluto por adsorção molecular e permuta iônica depende do pH da solução (Caetano et al., 2009). A troca de íons é reconhecida como uma alternativa para a remoção e recuperação de poluentes inorgânicos, caracteriza-se pelo fácil manuseio, baixos custos operacionais e permite a reutilização e purificação do efluente (Martins et al., 2017). A troca iônica é amplamente utilizada para o tratamento de água tanto em sistemas de tratamento de águas industriais quanto municipais. 0 processo oferece diversas vantagens em relação a outros métodos de tratamento. É ecológico, pode fornecer alta vazão de água tratada e tem baixo custo de manutenção. As indústrias, de uma maneira geral, têm cada vez mais utilizado as chamadas resinas trocadoras iônicas, pois elas ajudam a reduzir os resíduos líquidos gerados pelos processos químicos e também fazem com que haja possibilidade de reuso da água industrial.

\section{Processos oxidativos avançados (POAs)}

Os POAs são métodos favoráveis ambientalmente e eficientes métodos de desinfecção (Ginnnakis et al., 2017). Estes processos têm se destacado, por serem métodos extremamente eficiente na redução dos impactos ambientais, no desenvolvimento de tecnologias de tratamento de águas residuais. A eficácia dos POAs depende da geração de radicais livres reativos, sendo o mais importante, o radical hidroxila $\left(\mathrm{OH}^{*}\right.$ ) (Araújo et al. 2016). Dentre os POAs químicos, o processo Fenton é um do que mais se destaca (Araújo et al., 2016). Este processo utiliza íons ferrosos $\left(\mathrm{Fe}^{2+}\right)$ ou férricos $\left(\mathrm{Fe}^{3+}\right)$ como catalisadores da reação que em meio ácido, promove a decomposição de $\mathrm{H}_{2} \mathrm{O}_{2}$ gerando os radicais hidroxilhas $\left(\mathrm{HO}^{*}\right)$ que irá decompor as diferentes matérias orgânicas (Brillas et al., 2009).

Os POAs fotoquímicos são tecnologias simples e mais eficientes se comparados aos POAs químicos, com potencial para destruir uma alta porcentagem dos poluentes presentes. Quando se associa irradiação UV com agentes oxidantes fortes como, peróxido de hidrogênio $\left(\mathrm{H}_{2} \mathrm{O}_{2}\right)$, ozônio $\left(\mathrm{O}_{3}\right)$ e a catálise com dióxido de titânio $\left(\mathrm{TiO}_{2}\right)$, se gera diversos tipos de POAs fotoquímicos capazes de degradar ou destruir poluentes, como por exemplo o processo de Foto-Fenton (Araújo et al., 2016). Este processo consiste no Fenton assistido por irradiação UV que induz a redução catalítica em soluções aquosas de $\mathrm{H}_{2} \mathrm{O}_{2} \mathrm{e}$ de $\mathrm{Fe}^{3+}$ (íons férricos) a $\mathrm{Fe}^{2+}$ (íons ferrosos). Também ocorre a decomposição direta de moléculas de $\mathrm{H}_{2} \mathrm{O}_{2}$ em radicais hidroxila como no processo $\mathrm{H}_{2} \mathrm{O}_{2} / \mathrm{UV}$. Para a redução de custos para a realização do Foto-Fenton, a irradiação pode ser obtida através de luz solar (Araújo et al., 2016).

A fotólise também é um processo fotoquímico eficiente. Este processo pode ocorrer naturalmente, sendo uma alternativa para degradar compostos orgânicos tóxicos. $\mathrm{O} \mathrm{H}_{2} \mathrm{O}_{2}$ pode ser fotolisado por irradiação, ocasionando a quebra da ligação 0-0 da molécula de $\mathrm{H}_{2} \mathrm{O}_{2}$ gerando radicais hidroxila $(\mathrm{OH})$ que age na degradação de espécies orgânicas (Hernandez et al., 2002). Outro exemplo de POA Fotoquímico é a fotocatálise heterogênea, que consiste em uma 
tecnologia de foto-indução que atua com irradiação sobre o catalisador sólido semicondutor, em geral com dióxido de titânio $\left(\mathrm{TiO}_{2}\right)$ (Araújo, 2014).

A oxidação sonoquímica também é um exemplo de POA. É baseada na utilização de irradiação ultrassom (US) a fim de criar um ambiente oxidativo. Com a irradiação de ultrassom os radicais hidroxila são formados através de diferentes gases e em combinação com outros processos $\left(\mathrm{O}_{3} / \mathrm{US}, \mathrm{H}_{2} \mathrm{O}_{2} / \mathrm{US}\right.$ e fotocatálise/US) (Adewuyi, 2001). A sonólise é um processo fundamentado especialmente na cavitação acústica, abrangendo a formação, o crescimento e o colapso implosivo de bolhas em um líquido (Araújo et al., 2016).

Os processos com associações de POAs podem ser em determinados casos muito eficientes. Os processos combinados de ozônio com ultrassom $\left(\mathrm{O}_{3} /\right.$ US $)$, peróxido de hidrogênio com ultrassom $\left(\mathrm{H}_{2} \mathrm{O}_{2} / \mathrm{US}\right)$ ou ultrassom com fotocatálise (fotocatálise/US) ocasionam um aumento expressivo da taxa de geração de radicais livres, por exemplo (Chakinala et al., 2009). Outra Associação possível é o processo Sono-Fenton, sendo este a junção da radiação ultrassônica com o processo Fenton, aonde a decomposição de $\mathrm{H}_{2} \mathrm{O}_{2}$ sob radiação ultrassônica promove a aceleração da degradação dos contaminantes através da maior concentração de hidroxila (Chakinala et al., 2009).

Com relação aos Processos

Eletroquimicos Oxidativos Avançados, estes além de serem eficiente com a remoção de poluentes orgânicos persistentes, apresentam várias características de relevância ambiental, como reatores compactos de menor espaço físico, nenhuma exigência adicional de produtos químicos auxiliares, não produzem fluxos de resíduos secundários que requerem tratamento adicional, facilmente combinado com outras tecnologias de tratamento de água convencionais, facilmente automatizado e custos acessíveis para implantação (Garcia-
Segura et al., 2017). Os POAs eletroquímicos (POAEs) consistem na transferência de elétrons, fazendo com que o processo seja uma forma eficiente e sem poluentes de produção da radical hidroxila $(\mathrm{OH})$ para destruição de uma grande variedade de poluentes. Este processo de degradação pode ocorrer diretamente (processo de oxidação anódica) ou indiretamente (processo eletro-Fenton) (Araújo, 2014). O processo fotoeletro-Fenton (PFE) envolve o tratamento de soluções sob condições de eletro-Fenton com utilização simultânea de radiação ultravioleta (UV) ou luz visível, durante ou após a eletrólise, para acelerar a taxa de mineralização dos poluentes (Araújo, 2014).

Os POAs são técnicas que estão em desenvolvimento para a melhoria do tratamento de águas residuais. Contudo, sua eficiência na oxidação dos compostos poluentes, em especial compostos tóxicos persistentes, e a necessidade de novas formas mais eficientes de tratamento faz com que estudos sejam desenvolvidos afim de viabilizar este processo. Em geral estes estudos buscam diminuir o custo da operação, desenvolver materiais catalíticos e reatores em escalas industriais.

\section{Ozonização}

A ozonização é uma técnica de oxidação química que tem como agente oxidante o ozônio $\left(\mathrm{O}_{3}\right)$, oxidante energético, produzido a partir do ar, do oxigênio puro ou da água altamente pura (Hoigné, et al., 1976; Amado-Piña et al., 2017). Muito utilizado em processos de degradação de compostos orgânicos, pode reagir através de mecanismo direto (reação eletrofílica ou por cicloadição) ou mecanismo indireto (através do radical livre hidroxil formado a partir da decomposição do ozônio) Equações 5 e 6 (Fioreze, 2014; Freire et al., 2000; Masten e Davies, 1994).

Por apresentar-se sob forma gasosa em condições ambientais normais, o ozônio é altamente reativo e 
instável, o que significa que não pode ser transportado ou armazenado, tendo que ser produzido no local de aplicação (Andreoli, 2014; El-Taliawy, 2017). 0 alto interesse no uso de ozônio para desinfecção deve-se ao seu poder oxidante e a possibilidade de gerar radicais hidroxílicos não seletivos, tratase de uma das substâncias de mais alto potencial de oxidação (Andrioli, 2014; Gomes, 2017). Sob condições de tratamento de água, ele realiza funções de oxidação, precipitação e sanitização. Reage com sítio ativo de alta densidade eletrônica, como ligações não saturadas e aromáticas (El-Taliawy, 2017; Gomes, 2017). 0 ozônio pode atuar como agente desinfectante, biocida ou oxidante para remover poluentes orgânicos e como agente pré ou pós-tratamento para auxiliar outros processos, como a floculação ou sedimentação (Amado-Piña et al., 2017).

Pesquisas relataram que as características do $\mathrm{pH}$ e das águas residuais têm um efeito significativo na ozonização, bem como a combinação de processos de oxidação avançados e processos biológicos para o tratamento de águas residuais industriais sintéticas e reais (Oller et al., 2011; Sivagami et al., 2017; Srinivasan et al., 2009). Quando se trata da ozonização, deve-se considerar que é uma tecnologia promissora para o tratamento de contaminantes persistentes e tóxicos, que tem grande aplicação para o tratamento de águas residuárias, devido à grande eficiência na rápida mineralização de espécies químicas de importância ambiental. Porém, ainda é considerada uma tecnologia de alto custo devido algumas limitações como a necessidade de ser produzido in situ, não podendo ser armazenado ou transportado, sendo assim são necessários maiores estudos no aperfeiçoamento dessa tecnologia.

\section{Precipitação química}

A precipitação química é um dos processos comumente empregados e convencionais para o tratamento de efluentes, aplicada, principalmente para tratar águas residuárias que contém altas concentrações de metais ou sulfatos (Silva et al., 2002; Benatti et al., 2009; Dou et al., 2017). 0 processo ocorre quando determinado produto químico reage com os íons de metal pesado, ocasionando a formação de um sólido denominado precipitado (Zhao et al., 2016). A precipitação química pode ser obtida em quatro estágios, sendo precipitação, coagulação, floculação e separação (Henze et al., 1996).

0 processo de precipitação química utilizando hidróxido é simples e amplamente utilizado. A reação química em que os íons de metais pesados formam precipitação de hidróxido se associa ao $\mathrm{pH}$ da solução aquosa. Baseando-se na gama de $\mathrm{pH}$ estreita da solubilidade de vários hidróxidos metálicos, a remoção de metais pesados de águas residuais pode ser conseguida controlando o pH com baixo custo e facilidade de entrega de produtos químicos. Descobriu-se que os metais pesados bivariados, como $\mathrm{Cu}, \mathrm{Mn}$, podem facilmente formar precipitação de hidróxido e serem removidos das águas residuais (Alvarez-Ayuso et al., 2003). A precipitação utilizando sulfeto é outro processo eficiente para a remoção de metais pesados de águas residuais, onde a reação entre $\mathrm{H}_{2} \mathrm{~S}$ e íons de metais é o mecanismo predominante para realização do processo (Xie et al., 2012).

Em comparação com o processo de precipitação com hidróxido, os precipitados de sulfetos metálicos resultam na obtenção de um alto grau de remoção de metais em uma ampla gama de $\mathrm{pH}$. Além de lodo de sulfeto de metal ser mais fácil de tratar devido sua espessura.

Outra maneira de precipitação ocorre quando o cloreto de bário reage com sulfato de sódio, o sulfato de bário insolúvel é formado como um precipitado. Dessa forma os íons sulfato podem ser removidos da solução através da adição de uma quantidade adequada de cloreto de bário (Kartic et al., 2018). 0 
método de precipitação é amplamente utilizado para a remoção de aníons inorgânicos, como por exemplo, na remoção de amônia, fosfato e flúor de águas residuais de semicondutores através do uso de sais de magnésio (Huang et al., 2017). Sais de cálcio podem ser utilizados na precipitação química para remoção de fosfato e flúor de transistores de filme fino (TFT-LCD). Águas residuais com alto teor de flúor originadas em indústrias de fertilizantes podem ser removidos pela adição de cloreto de cálcio (Liu e Liu, 2016). O método de precipitação química, apresenta como principal desvantagem a geração de grandes quantidades de lodo, que necessitam de uma destinação final adequada devido ao potencial de toxicidade (Duo et al, 2017).

\section{Conclusões}

Através desta revisão referente aos diferentes sistemas de tratamento físico-químicos de remoção de contaminantes em efluentes foi possível verificar que existem diferentes tecnologias que podem ser aplicadas, entretanto cada uma apresenta limitações quanto ao seu uso, que devem ser avaliadas em virtude das características do efluente que se busca tratar, bem como das finalidades a qual estas águas residuárias serão aplicadas ou destinadas após o tratamento.

\section{Conflito de interesses}

Os autores declaram não haver conflito de interesses.

\section{Referências}

Abdel-Raouf, N.; Al-Homaidan, A. A.; Ibraheem, I. B. M. Microalgae and wastewater treatment. Saudi Journal of Biological Sciences, v. 19, n. 3, p. 257-275, 2012. https://doi.org/10.1016/j.sjbs.2012.04.005

Adewuyi, Y.G. Sonochemistry: Environmental science and engineering applications. Industrial \& Engineering Chemistry
Research, v. 40, n. 22, p. 4681-4715, 2001. https://doi.org/10.1021/ ie010096l

Aguiar, A. O.; Andrade, L. H.; Ricci, B. C.; Pires, W. L.; Miranda, G. A.; Amaral, M. C. S. Gold acid mine drainage treatment by membrane separation processes: An evaluation of the main operational conditions. Separation and Purification Technology, v. 170, p. 360-369, 2016. https://doi.org/10.1016/j.seppur. 2016.07.003

Aguiar, A.; Andrade, L.; Grossi, L.; Pires, W.; Amaral, M. Acid mine drainage treatment by nanofiltration: A study of membrane fouling, chemical cleaning, and membrane ageing. Separation and Purification Technology, v. 192, p.185-195, 2018. https://doi.org/ 10.1016/j.seppur.2017.09.043

Al-Shannag, M.; Al-Qodah, Z.; Bani-Melhem, K.; Qtaishat, M. R.; Alkasrawi, M. Heavy metal ions removal from metal plating wastewater using electrocoagulation: Kinetic study and process performance. Chemical Engineering Journal, v. 260, p. 749-756, 2015. https://doi.org/10.1016/j.cej.2014.09.035

Alvarez-Ayuso, A. E.; García-Sánchez, A.; Querol, X. Purification of metal electroplating wastewaters using zeolites. Water Research, v. $37, \quad$ n. $20, \quad$ p. $4855-4862,2003$. https://doi.org/10.1016/j.watres.2003.08.00 9

Alvarino, T.; Suarez, S.; Lema, J.; Omil, F. Understanding the sorption and biotransformation of organic micropollutants in innovative biological wastewater treatment technologies. Science of the Total Environment, v. 615, p. 297-306, 2018. https://doi.org/10.1016/j.scitotenv.2017.09. 278

Amado-Piña, D. A.; Roa-Morales, G.; BarreraDíaz, C.; Balderas-Hernandez, P.; Romero, R.; Campo, E. M.; Natividad, R. Synergic effect of ozonation and electrochemical methods on oxidation and toxicity reduction: Phenol degradation. Fuel, v. 198, p. 82-90, 2017. https://doi.org/10.1016/j.fuel.2016.10.117

Amaral, C. N. R.; Feiteira, F. N.; Cruz, R. C.; Cravo, V. O.; Cassella, R. J.; Pacheco, W. F. Removal of basic violet 3 dye from aqueous media using a steel industry residue as solid phase. Journal of Environmental Chemical Engineering, v. 4, p. 4148-4193, 2016. https://doi.org/10.1016/j.jece.2016.09.023

Andrade, L. H.; Aguiar, A. O.; Pires, W. L. Miranda, G. A.; Teixeira, L. P. T.; Almeida, G. C. C.; Amaral, M. C. S. Nanofiltration and reverse 
osmosis applied to gold mining effluent treatment and reuse. Brazilian Journal of Chemical Engineering, v. 34, n. 1, p. 93-107, 2017b. https://doi.org/10.1590/01046632.20170341s20150082

Andrade, L. H.; Ricci, B. C.; Grossi, L. B.; Pires, W. L.; Aguiar, A. O.; Amaral, M. C. S. Nanofiltration applied in gold mining effluent treatment: Evaluation of chemical cleaning and membrane stability. Chemical Engineering Journal, v. 323, p. 545-556, 2017a. https://doi.org/10.1016/j.cej.2017. 04.116

Andrioli, E.; Mella, B.; Gutterres, M. A tecnologia de ozonização no tratamento de efluentes de curtume. Anais do II Congresso Brasileiro de Engenharia Química, Florianópolis, p. 1-8, 2014.

Araújo, K. S.; Antonelli, R.; Gaydeczka, B.; Granato, A. C.; Malpass, G. R. P. Processos oxidativos avançados: uma revisão de fundamentos e aplicações no tratamento de águas residuais urbanas e efluentes industriais. Revista Ambiente \& Água, v. 11, n. 2 , p. 387-401, 2016. https://doi.org/ 10.4136/ambi-agua.1862

Araújo, K. S.; Malpass, G. R. P.; Urias, P. M.; Cunha, P. C. R. Processos oxidativos avançados: fundamentos e aplicações no tratamento de águas residuais e efluentes industriais. Anais do V Congresso Brasileiro de Gestão Ambiental, Belo Horizonte, IBEAS, 2014.

Babaei, A. A.; Kakavandi, B.; Rafiee, M.; Kalantarhormizi, F.; Purkaram, I.; Ahmadi, E.; Esmaeili, S. Comparative treatment of textile wastewater by adsorption, Fenton, UVFenton, and US-Fenton using magnetic nanoparticles-functionalized carbon (MNPs@c). Journal of Industrial and Engineering Chemistry, v. 56, p. 163-174, 2017. https://doi.org/10.1016/j.jiec.2017. 07.009

Babilas, D.; Dydo, P. Selective zinc recovery from electroplating wastewaters by electrodialysis enhanced with complex formation. Separation and Purification Technology, v. 192, p. 419-428, 2018. https://doi.org/10.1016/j.seppur.2017.10.01 3

Badruzzaman, M.; Oppenheimer, J.; Adham, S.; Kumar, M. Innovative beneficial reuse of reverse osmosis concentrate using bipolar membrane electrodialysis and electrochlorination processes. Journal of
Membrane Science, v. 326, n. 2, p. 392-399, 2009. https://doi.org/10.1016/j.memsci. 2008.10.018

Bassala, H. D.; Dedzo, G. K.; Bememba, C. B. N.; Seumo, P. M. T.; Dazie, J. D.; Nanseu-Njiki, C. P.; Ngameni, E. Investigation of the efficiency of a designed electrocoagulation reactor: Application for dairy effluent treatment. Process Safety and Environmental Protection, v. 111, p.122-127, 2017. https://doi.org/10.1016/j.psep.2017.07.002

Bell, E. A.; Poynor, T. E.; Newhart, K. B.; Regnery, J.; Coday, B. D.; Cath, T. Y. Produced water treatment using forward osmosis membranes: Evaluation of extended-time performance and fouling. Journal of Membrane Science, v. 525, p. 77-88, 2017. https://doi.org/10.1016/j.memsci.2016.10.0 32

Ben, W.; Wang, J.; Cao, R.; Yang, M.; Zhang, Y. Qiang, Z. Distribution of antibiotic resistance in the effluents of ten municipal wastewater treatment plants in China and the effect of treatment processes. Chemosphere, v. 172, p. 392-398, 2017. https://doi.org/10.1016/ j.chemosphere.2017.01.041

Benatti, C. T.; Tavares, C. R. G.; Lenzi, E. Sulfate removal from waste chemicals by precipitation. Journal of Environmental Management, v. 90, n. 9, p. 504-511, 2009. https://doi.org/10.1016/j.jenvman.2007.12. 006

Bengani-Lutz, P.; Zaf, R. D.; Culfaz-Emecem, Z.; Asatekin, A. Extremely fouling resistant zwitterionic copolymer membranes with $\sim 1 \mathrm{~nm}$ pore size for treating municipal, oily and textile wastewater streams. Journal of Membrane Science, v. 543, p. 184-194, 2017. https://doi.org/10.1016/j.memsci. 2017.08.058

Bernardi, F.; Zadinelo, I. V.; Alves, H. J.; Meurer, F.; Santos, L. D. Chitins and chitosans for the removal of total ammonia of aquaculture effluents. Aquaculture, v. 483, p. 203-201, 2018. https://doi.org/10.1016/ j.aquaculture.2017.10.027

Bortoluzzi, A. C.; Faitão, J. A.; Di Luccio, M.; Dallago, R. M.; Steffens, J.; Zabot, G. L.; Tres, M. V. Dairy wastewater treatment using integrated membrane systems. Journal of Environmental Chemical Engineering, v. 5, n. 5, p. 4819-4827, 2017. https://doi.org/ 10.1016/j.jece.2017.09.018

Brillas, E.; Sires, I.; Oturan, M. A. Electrofenton process and related electrochemical 
technologies based on Fenton's reaction chemistry. Chemical Reviews, v. 109, v. 12, p. 6570-6631, 2009. https://doi.org/ $10.1021 / \mathrm{cr} 900136 \mathrm{~g}$

Caetano, M.; Valderrama, C.; Farran, A.; Cortina, L. J. Phenol removal from aqueous solution by adsorption and ion exchange mechanisms onto polymeric resins. Journal of Colloid and Interface Science, v. 338, n. 2, p. 402-409, 2009. https://doi.org/ 10.1016/j.jcis.2009.06.062

Centenaro, G. S. N. M.; Facin, B. R.; Valério, A.; Souza, A. A. U.; Silva, A.; Oliveira, J. V.; Oliveira, D. Application of polyurethane foam chitosan-coated as a low-cost adsorbent in the effluent treatment. Journal of Water Process Engineering, v. 20, p. 201-206, 2017. https://doi.org/10.1016/j.jwpe.2017. 11.008

Chakinala, A. G.; Gogate, P. R.; Burgess, A. E.; Bremner, D. H. Industrial wastewater treatment using hydrodynamic cavitation and heterogeneous advanced Fenton processing. Chemical Engineering Journal, $\begin{array}{llll}\text { v. } 152, & \text { n. } 2 / 3, & \text { p. } 498-502,\end{array}$ https://doi.org/10.1016/j.cej.2009.05.018

Chen, G. Electrochemical technologies in wastewater treatment. Separation and Purification Technology, v. 38, n. 1, p.11-41, 2004. https://doi.org/10.1016/j.seppur. 2003.10.006

Chou, S.; Shi, L.; Wang, R.; Tang, C. Y.; Qiu, C.; Fane, A. G. Characteristics and potential applications of a novel forward osmosis hollow fiber membrane. Desalination, $\begin{array}{lll}\text { v. } 261, & \text { n. } 3, \quad \text { p. } 365-372, & 2010 .\end{array}$ https://doi.org/10.1016/j.desal.2010.06.027

Cingolani, D.; Eusebi, A. L.; Battistoni, P. Osmosis process for leachate treatment in industrial platform: Economic and performances evaluations to zero liquid discharge. Journal of Environmental Management, v. 203, part 2, p. 782-790, 2017. https://doi.org/10.1016/j.jenvman. 2016.05.012

Combernoux, N.; Schrive, L.; Labed, V.; Wyart, Y.; Carretier, E.; Moulin, P. Treatment of radioactive liquid effluents by reverse osmosis membranes: From lab-scale to pilotscale. Water Research, v. 123, p. 311-320, 2017. https://doi.org/10.1016/j.watres. 2017.06.062

Daneshvar, N.; Oladegaragoze, A.; Djafarzadeh, N. D. Decolorization of basic dye solutions by electrocoagulation: An investigation of the effect of operational parameters. Journal of Hazardous Materials, v. 129, n. 1/3, p. 116-122, 2006. https://doi.org/10.1016/j.jhazmat.2005.08.0 33

Delgado, L. F.; Charles, P.; Glucina, K.; Morlay, C. The removal of endocrine disrupting compounds and cyanobacterial toxins during drinking water preparation using activated carbon: A review. Science of The Total Environmental, v. 435-436, n. 1, p. 509-525, 2012. https://doi.org/10.1016/j.scitotenv. 2012.07.046

Dou, W.; Zhou, Z.; Jiang, L.; Jiang, A.; Huang, R.; Tian, X.; Zhang, W.; Chen, D. Sulfate removal from wastewater using ettringite precipitation: Magnesium ion inhibition and process optimization. Journal of Environmental Management, v. 196, p. 518526, 2017. https://doi.org/10.1016/ j.jenvman.2017.03.054

Duong, P. H. H.; Chung, T.-S. Application of thin film composite membranes with forward osmosis technology for the separation of emulsified oil-water. Journal of Membrane Science, v. $452, \quad$ p. 117-126, 2014. https://doi.org/10.1016/j.memsci.2013.10.0 30

Edokpayi, J. N.; Odiyo, J. O.; Durowoju, O. D. Impact of wastewater on surface water quality in developing countries: A case study of South Africa. In: Tutu, H. Water quality. London: Intech, 2017. p. 401-416.

El-Taliawy, H.; Ekblad, M.; Nilsson, F.; Hagman, M.; Paxeus, N.; Jönsson, K.; Cimbritz, M.; Jansen, J. L. C.; Bester, K. Ozonation efficiency in removing organic micropollutants from wastewater with respect to hydraulic loading rates and diferente wastewaters. Chemical Engineering Journal, v. 325, p. 310-321, 2017. https://doi.org/10.1016/j.cej.2017.05.019

Fernandes, A.; Pacheco, M. J.; Ciríaco, L.; Lopes, A. Review on the electrochemical processes for the treatment of sanitary landfill leachates: Present and future. Applied Catalysis B: Environmental, v. 176-177, p. 183-200, 2015. https://doi.org/10.1016/j.apcatb.2015.03.05 2

Fioreze, M.; Santos, E. P.; Schmachtenberg, N. Processos oxidativos avançados: fundamentos e aplicação ambiental. Revista Eletrônica em Gestão, Educação e Tecnologia Digital, v. 18, n. 1, p. 79-91, 
2014. https://doi.org/10.5902/22361170 10662

Fosso-Kankeu, E.; Mittal, H.; Waanders, F.; Ray, S.S. Thermodynamic properties and adsorption behavior of hydrogel nanocomposites for cadmium removal from mine effluents. Journal of Industrial and Engineering Chemistry, v. 48, p. 151-161, 2017. https://doi.org/10.1016/j.jiec.2016. 12.033

Freire, R. S.; Kunz, A.; Durán, N. Some chemical and toxicological aspects about paper mill effluent treatment with ozone. Environmental Technology, v. 21, n. 6, p. 717-721, 2000. https://doi.org/10.1080/ 09593332108618088

Gao, W.; Fatehi, P. Fly ash based adsorbent for treating bleaching effluent of kraft pulping process. Separation and Purification Technology, v. 195, p. 60-69, 2018 . https://doi.org/10.1016/j.seppur. 2017.12.002

Gao, Y.; Fang, Z.; Liang, P.; Huang, X. Direct concentration of municipal sewage by forward osmosis and membrane fouling behavior. Bioresource Technology, v. 247, p. 730-735, $2018 . \quad$ https://doi.org/ 10.1016/j.biortech.2017.09.145

Garcia-Segura, S.; Ocon, J.D.; Chong, M. N. Electrochemical oxidation remediation of real wastewater effluents: A review. Process Safety and Environment Protection, v. 113, p. 48-67, 2017. https://doi.org/10.1016/ j.psep.2017.09.014

Gomes, J.; Costa, R.; Quinta-Ferreira, R. M.; Martins, R. C. Application of ozonation for pharmaceuticals and personal care products removal from water. Science of The Total Environment, v. 586, p. 265-283, 2017. https://doi.org/10.1016/j.scitotenv.2017.01. 216

Hemmati, F.; Norouzbeigi, R.; Sarbishedh, F.; Shayesteh, H. Malachite green removal using modified sphagnum peat moss as a low-cost biosorbent: Kinetic, equilibrium and thermodynamic studies. Journal of the Taiwan Institute of Chemical Engineers, v. 58, p. 485-489, 2016. https://doi.org/ 10.1016/j.jtice.2015.07.004

Henze, M.; Harremoes, P.; Arvin, E.; Jansen, J. L. C. Wastewater treatment: Biological and chemical processes. 2 ed. New York: Springer, 1996.

Hernandez, R.; Zappi, M.; Colluci, F.; Jones, R. Comparing the performance of various advanced oxidation process for treatment of acetone contaminated water. Journal Hazardous Materials, v. 92, n. 1, p. 33-50, $2002 . \quad$ https://doi.org/10.1016/S03043894(01)00371-5

Hickenbottom, K. L.; Hancock, N. T.; Hutchings, N. R.; Appleton, E. W.; Baeudry, E. G.; Xu, P.; Cath, T. Y. Forward osmosis treatment of drilling mud and fracturing wastewater from oil and gas operations. Desalination, v. 312, p. 60-66, 2013. https://doi.org/10.1016/j.desal.2012.05.037

Hoigné, J.; Bader, H. The role of hydroxyl radical reactions in ozonation processes inaqueous solutions. Water Research, v. 10, n. 5, p. 377-386, 1976. https://doi.org/ 10.1016/0043-1354(76)90055-5

Holt, P. K.; Barton, G. W.; Wark, M.; Mitachell, C. A. A quantitative comparison between chemical dosing and electrocoagulation. Colloids and Surfaces A: Physicochemical Engineering Aspects, v. 211, n. 2/3, p. 233248, 2002. https://doi.org/10.1016/S09277757(02)00285-6

Hudaib, B.; Gomes, V.; Shi, J.; Zhou, C.; Liu, Z. Poly (vinylidene fluoride)/polyaniline/ MWCNT nanocomposite ultrafiltration membrane for natural organic matter removal. Separation and Purification Technology, v. 190, p. 143-155, 2018. https://doi.org/10.1016/j.seppur.2017.08.02 6

Judd, S.J. The status of industrial and municipal effluent treatment with membrane bioreactor technology. Chemical Engineering Journal, v. 305, p. 37-45, 2016. https://doi.org/10.1016/j.cej.2015.08.141

Juholin, P.; Kääriäinen, M.-L.; Riihimäki, M.; Sliz, R.; Aguirre, J. L.; Pirilä, M.; Fabritius, T.; Cameron, D.; Keiski, R. L. Comparison of ALD coated nanofiltration membranes to unmodified commercial membranes in mine wastewater treatment. Separation and Purification Technology, v. 192, p. 69-77, 2018. https://doi.org/10.1016/j.seppur. 2017.09.005

Jung, K.; Hwang, M.; Park, D.; Ahn, K. Combining fluidized metal-impregnated granular activated carbon in threedimensional electrocoagulation system: feasibility and optimization test of color and COD removal from real cotton textile wastewater. Separation and Purification Technology, v. 146, p.154-167, 2015. 
https://doi.org/10.1016\%2Fj.seppur.2015.0 3.043

Karanfil, T.; Kilduff, J. Role of granular activated carbon surface chemistry on the adsorption of organic compounds. Priority pollutants. Environmental Science Technology, v. 33, n. 18, p. 3217-3224, 1999. https://doi.org/10.1021/es981016g

Kartic, D. N.; Narayana, B. C. A.; Arivazhagan, M. Removal of high concentration of sulfate from pigment industry effluent by chemical precipitation using barium chloride: RSM and ANN modeling approach. Journal of Environmental Management, v. 206, p. 6976, 2018. https://doi.org/10.1016/ j.jenvman.2017.10.017

Kumar, P.; Sharma, N.; Ranjan, R.; Kumar, S.; Bhat, Z.F.; Jeong, D. K. Perspective of membrane technology in dairy industry: A review. Asian-Australasian Journal of Animal Sciences, v. 26, n. 9, p. 1347-1358, 2013. https://doi.org/10.5713/ajas.2013. 13082

Li, N.; Whang, P.; Liu, Q.; Cao, H. Microwave enhanced chemical reduction process for nitrite-containing wastewater treatment using sulfaminic acid. Journal of Environmental Sciences, v. 22, n. 1, p. 5651, 2010. https://doi.org/10.1016/S10010742(09)60074-7

Lin, J.; Ye, W.; Baltaru, M.; Tang, Y. P.; Bernstein, N. J.; Gao, P.; Balta, S.; Vlad, M.; Volodin, A.; Sotto, A.; Luis, P.; Zydney, A. L.; Bruggen, B. V. Tight ultrafiltration membranes for enhanced separation of dyes and $\mathrm{Na}_{2} \mathrm{SO}_{4}$ during textile wastewater treatment. Journal of Membrane Science, v. 514 , p. $217-228,2016$. https://doi.org/ 10.1016/j.memsci.2016.04.057

Liu, C.; Mao, H.; Zheng, J.; Zhang, S. Tight ultrafiltration membrane: Preparation and characterization of thermally resistant carboxylated cardo poly (arylene ether ketone)s (PAEK-COOH) tight ultrafiltration membrane for dye removal. Journal of Membrane Science, v. 530, p. 1-10, 2017. http://doi.org/10.1016/j.memsci.2017.02.00 5

Liu, G. C.; Yu, S. L.; Yang, H. J.; Hu, J.; Zhang, Y.; He, B.; Li, L.; Liu, Z. Y. Molecular mechanisms of ultrafiltration membrane fouling in polymer-flooding wastewater treatment: Role of ions in polymeric fouling. Environmental Science \& Technology, v. $50, \quad$ n. $3, \quad$ p. $1393-1402, \quad 2016$. https://doi.org/10.1021/acs.est.5b04098

Luján-Facundo, M. J.; Soler-Cabezas, J. L. Mendoza-Roca, J. A.; Vicent-Vela, M. C.; BesPiá, A.; Doñate-Hernández, S. A study of the osmotic membrane bioreactor process using a sodium chloride solution and an industrial effluent as draw solutions. Chemical Engineering Journal, v. 322, p. 603-610, 2017. https://doi.org/10.1016/j.cej.2017. 04.062

Luo, A.; Lior, N. Study of advancement to higher temperature membrane distillation. Desalination, v. 419, p.88-100, 2017. https://doi.org/10.1016/j.desal.2017.05.020

Marcogliese, D. J.; Blaise, C.; Cyr, D.; Lafontaine, Y.; Fournier; M.; Gagné, F.; Gagnon, C.; Hudon, C. Effects of a major municipal effluent on the St. Lawrence River: A case study. AMBIO, v. 44, n. 4, p. 257-274, 2015. https://doi.org/10.1007/s13280-0140577-9

Martins, P. J. M.; Reis, P. M.; Martins, R. C.; Guando-Ferreira, L. M.; Quinta-Ferreira, R. M. Iron recovery from the Fenton's treatment of winery effluent using an ion-exchange resin. Journal of Molecular Liquids, v. 242, p. 505511, 2017. https://doi.org/10.1016/j.molliq. 2017.07.041

Masten, S. J.; Davies, S. H. R. The use of ozonation to degrade organic contaminants in wastewaters. Environmental Science Technology, v. 28, n. 4, p. 180-185, 1994. https://doi.org/10.1021\%2Fes00053a718

Mohammad, A. W.; Teow, Y. H.; Ang, W. L.; Chung, Y. T.; Oatley-Radcliffe, D. L.; Hilal, N. Nanofiltration membranes review: Recent advances and future prospects. Desalination, v. 356, p. 226-254, 2015. https://doi.org/10.1016/j.desal.2014.10.043

Mollah, Y. M. A.; Schennach, R.; Parga, J. R.; Cocke, D. L. Electrocoagulation (EC): Science and applications. Journal of Hazardous Materials, v. 84, n. 1, p. 29-41, 2001. https://doi.org/10.1016/S03043894(01)00176-5

Noor, S. F. M.; Ahmad, N.; Khattak, M. A.; Khan, M. S.; Mukhtar, A.; Kazi, S.; Badshah, S.; Khan, R. Application of Sayong Ball Clay Membrane Filtration for Ni (II) removal from industrial wastewater. Journal of Taibah University for Science, v. 11, n. 6, p. 949954, 2017. https://doi.org/10.1016/j.jtusci. 2016.11.005 
Oller, I.; Malato, S.; Sánchez-Pérez, J. A. Combination of advanced oxidation processes and biological treatments for wastewater decontamination: A review. Science of the Total Environment, v. 409, n. 20, p. 4141-4166, 2011. https://doi.org/ 10.1016/j.scitotenv.2010.08.061

Ortega, V. M. D.; Ochando-Pulido, J. M.; Martínez-Ferez, A. Ion exchange system for the final purification of olive mill wastewater: Performance of model vs. real effluent treatment. Process Safety and Environmental Protection, v. 103, p. 308314, 2016. https://doi.org/10.1016/j.psep. 2016.02.004

Pintar, A.; Batista, J.; Levec, J. Integrated ion exchange/catalytic process for efficient removal of nitrates from drinking water. Chemical Engineering Science, v. 56, n. 4, p. 1551-1559, 2001. https://doi.org/ 10.1016/S0009-2509(00)00382-1

Rafraf, I. D.; Lekunberri, I.; Sánchez-Melsió, A.; Aouni, M.; Borrego, C. M.; Balcázar, J. L. Abundance of antibiotic resistance genes in five municipal wastewater treatment plants in the Monastir Governorate, Tunisia. Environmental Pollution, v. 219, p. 353358, 2016. https://doi.org/10.1016/j.envpol. 2016.10.062

Rastgar, M.; Shakeri, A.; Bozorg, A.; Salehi, H.; Saadattalab, V. Impact of nanoparticles surface characteristics on pore structure and performance of forward osmosis membranes. Desalination, v. 421, p. 179-189, 2017. https://doi.org/10.1016/j.desal.2017.01.040

Ricci, B. C.; Ferreira, C. D.; Aguiar, A. O.; Amaral, M. C. S. Integration of nanofiltration and reverse osmosis for metal separation and sulfuric acid recovery from gold mining effluent. Separation and Purification Technology, v. 154, p.11-21, 2015. https://doi.org/10.1016/j.seppur.2015.08.04 0

Samartino, J. A.; Khayet, M.; García-Payo, M. C. Reuse of discarded membrane distillation membranes in microfiltration technology. Journal of Membrane Science, v. 539, p. 273-283, 2017. https://doi.org/10.1016/ j.memsci.2017.06.003

Silva, A. J.; Varesche, M. B.; Foresti, E.; Zaiat, M. Sulphate removal from industrial wastewater using a packed-bed anaerobic reactor. Process Biochemistry, v. 37, p. 927935, 2002. https://doi.org/10.1016/S00329592(01)00297-7
Sivagami, K.; Sakthivel, K. P.; Nambi, I. M. Advanced oxidation processes for the treatment of tannery wastewater. Journal of Environmental Chemical Engineering, v. 6, p. 3656-3663, 2017. https://doi.org/ 10.1016/j.jece.2017.06.004

Snyder, S. A.; Adham, S.; Redding, A. M.; Cannon, F. S.; De Carolis, J.; Oppenheimer, J.; Wert, E. C.; Yoon, Y. Role of membranes and activated carbon in the removal of endocrine disruptors and pharmaceuticals. Desalination, v. 202 , n. $1 / 3$, p. 156-181, 2007. https://doi.org/10.1016/j.desal.2005. 12.052

Srinivasan, S. V.; Rema, T.; Chitra, K.; Balakameswari, K. S.; Suthanthararajan, B.; Maheswari, B. U. Decolourisation of leather dye by ozonation. Desalination, v. 235, n. $1 / 3$, p. 88-92, 2009. https://doi.org/ 10.1016/j.desal.2007.07.032

Uslu, G.; Demirci, A.; Regan, J. M. Disinfection of synthetic and real municipal wastewater effluent by flow-through pulsed UV-light treatment system. Journal of Water Process Engineering, v. 10, p.89-97, 2016. https://doi.org/10.1016/j.jwpe.2016.02.004

Valverde, L. J.; Lucas, A.; Carmona, M.; Pérez, J. P.; Gozález, M.; Rodríguez, J. F. Minimizing the environmental impact of the regeneration process of an ion exchange bed charged with transition metals. Separation and Purification Technology, v. 49, n. 2, p. 167 173, 2006. https://doi.org/10.1016/j.seppur. 2005.09.009

Walker, S.; Narbaitz, R. M. Hollow fiber ultrafiltration of Ottawa River water: Floatation versus sedimentation pretreatment. Chemical Engineering Journal, v. 288 , p. 228-237, 2016. https://doi.org/ 10.1016/j.cej.2015.11.064

Wojtyniak, B.; Kołodziejczyk, J.; Szaniawska, D. Production of lactic acid by ultrafiltration of fermented whey obtained in bioreactor equipped with ZOSS membrane. Chemical Engineering Journal, v. 305, p. 28-36, 2016. https://doi.org/10.1016/j.cej.2016.01.048

Xie, L. P.; Fu, F. L.; Tang, B. Research progress in the treatment of complex heavy metal wastewater. Industrial Water Treatment, v. 32, p. 1-5, 2012. https://doi.org/10.1088/ 1755-1315/121/3/032022

Xie, M.; Price, W. E.; Nghiem, L. D. Rejection of pharmaceutically active compounds by forward osmosis: Role of solution $\mathrm{pH}$ and membrane orientation. Separation and 
Purification Technology, v. 93, p. 107-114, 2012. https://doi.org/10.1016/j.seppur. 2012.03.030

Yang, T.; Qiao, B.; Li, G.-C.; Yang, Q.-Y. Improving performance of dynamic membrane assisted by electrocoagulation for treatment of oily wastewater: Effect of electrolytic conditions. Desalination, v. 363, p. 134-143, 2015. https://doi.org/10.1016/ j.desal.2015.01.010

Yetilmezsoy, K.; Ilhan, F.; Sapci-Zengin, Z.; Sakar, S.; Gonullu, M. T. Decolorization and COD reduction of UASB pretreated poultry manure wastewater by electrocoagulation process: A post-treatment study. Journal of Hazardous Materials, v. 162, n. 1, p. 120-
132, 2009. https://doi.org/10.1016/ j.jhazmat.2008.05.015

Zhao, M.; Xu, Y.; Zhang, C.; Rong, H.; Zeng, G. New trends in removing heavy metals from wastewater. Applied Microbiology and Biotechnology, v. 100 , n. 15, p. 6509-6518, 2016.

Zielińska, M.; Galik, M. Use of Ceramic membranes in a membrane filtration supported by coagulation for the treatment of dairy wastewater. Water, Air, \& Soil Pollution, v. 228, p. 2-12, 2017. https://doi.org/10.1007/s11270-017-3365-

Informação da Licença: Este é um artigo Open Access distribuído sob os termos da Licença Creative Commons Attribution, que permite uso irrestrito, distribuição e reprodução em qualquer meio, desde que a obra original seja devidamente citada. 